\title{
Patricia Victorin, Ysaïe le Triste. Une Esthétique de la Confluence. Tours, Tombeaza, Vergers et Fontaines
}

\section{Richard Trachsler}

\section{(2) OpenEdition}

1 Journals

\section{Édition électronique}

URL : http://journals.openedition.org/studifrancesi/35667

DOI : 10.4000/studifrancesi.35667

ISSN : 2421-5856

Éditeur

Rosenberg \& Sellier

\section{Édition imprimée}

Date de publication : 1 juillet 2005

Pagination : 128-130

ISSN : 0039-2944

\section{Référence électronique}

Richard Trachsler, «Patricia Victorin, Ysaie le Triste. Une Esthétique de la Confluence. Tours, Tombeaza, Vergers et Fontaines », Studi Francesi [En ligne], 145 (XLIX | I) | 2005, mis en ligne le 30 novembre 2015, consulté le 19 avril 2021. URL : http://journals.openedition.org/studifrancesi/35667 ; DOI : https:// doi.org/10.4000/studifrancesi.35667

Ce document a été généré automatiquement le 19 avril 2021.

\section{(c)}

Studi Francesi è distribuita con Licenza Creative Commons Attribuzione - Non commerciale - Non opere derivate 4.0 Internazionale. 


\title{
Patricia Victorin, Ysaïe le Triste. Une Esthétique de la Confluence. Tours, Tombeaza, Vergers et Fontaines
}

\author{
Richard Trachsler
}

\section{RÉFÉRENCE}

PATRICIA VICTORIN, Ysaïe le Triste. Une Esthétique de la Confluence. Tours, Tombeaza, Vergers et Fontaines, Paris, Champion («Bibliothèque du XVe siècle», 63), 2002, pp. 501.

Dans ce volume de près de 500 pages, Patricia Victorin s'attache à faire découvrir à son lecteur Ysaïe le Triste, un des derniers romans arthuriens écrits en français avant le «renouveau» que connaîtra le genre dans les imprimés et les mises en prose. Contrairement, donc, à ce qui se passera quelques décennies plus tard, avec la vague des dérimages, Ysaïe le Triste propose une composition originale et non pas une oeuvre plus ancienne simplement mise au goût du jour. Cela ne veut pas dire, naturellement, que l'intrigue, les personnages, le style ne soient pas fortement ancrés dans la tradition littéraire antérieure, mais c'est indéniablement une œuvre composée à de nouveaux frais. En effet, l'auteur d'Ysaïe, tout en se coulant dans un moule en apparence bien connu, explore en même temps une terra incognita puisqu'il raconte les aventures d'Ysaïe, fils de Tristan et d'Yseut, et même celles de Marc, fils d'Ysaïe et par conséquent petit-fils des amants de Cornouaille. C'est donc, au moins pour certains aspects, un roman arthurien après Arthur, au même titre que le Perceforest, écrit à peu près vers la même époque, peut être considéré comme un roman arthurien avant Arthur. Concrètement, le romancier a recours, pour «meubler» son texte et peupler son univers, aux romans arthuriens cycliques, mais, de façon moins attendue, il fait appel aussi à la matière épique. Cette fusion lui est facilitée par le fait que la chanson de geste, à la fin du Moyen Age, emprunte largement la voie romanesque, créant de fait un grand genre narratif unique. C'est en raison de ces apports multiples, arthurien et épique, traditionnel et innovateur, que Patricia Victorin a choisi, pour sous-titre à son 
étude sur Ysaïe, «Esthétique de la Confluence». A travers les différentes parties de son livre, elle explore et commente cette "confluence» selon un plan qui permet d'en balayer tous les aspects: dans une première partie est examiné l'ancrage spatiotemporel par rapport au canevas arthurien, alors qu'une deuxième partie accomplit le même travail pour les personnages d'Ysaie. La troisième partie s'attache à «l'écriture de seconde main» et met en lumière les tentatives pour préserver la cohérence d'un roman menacé d'éclatement par 1'hétérogénéité de ses sources, et dans la dernière partie, Patricia Victorin aborde ouvertement le tiraillement entre une esthétique «omnivore», qui se veut totalisante, et une autre, plus proche du médaillon, qui fait une certaine place à la «mémoire miniaturisée». Outre l'index des critiques et celui des oeuvres citées, un troisième index permet de retrouver facilement les passages où il est question d'un motif, d'un personnage ou d'un procédé littéraire. Le style est alerte et Patricia Victorin a le sens de la formule, ce qui tient l'esprit du lecteur en éveil et le récompense quelque peu pour les efforts qui lui sont par endroits demandés pour pleinement tirer profit des pages de son guide. En effet, on sent encore ici et là que ce livre est issu d'une thèse. Il oublie parfois qu'il s'adresse à un lecteur qui ne fait pas partie d'un jury de thèse et qui ne s'est donc pas préparé exprès pour lire l'ouvrage en question: ainsi, il faut avoir une excellente maitrise d'Ysaïe le Triste pour comprendre, sans relire les passages correspondants dans l'édition, les fines observations de Patricia Victorin sur les songes (pp. 92-93) ou sur les insertions lyriques (pp. 248-74). A chaque fois, il aurait suffi de quelques phrases supplémentaires pour rappeler le contexte et les données et pour rendre plus directement exploitable le présent livre. Mais ce n'est là qu'une question de confort et n'enlève rien à l'intérêt du travail de Patricia Victorin. La plupart du temps, ses observations emportent la conviction et l'on trouvera judicieuses les lectures proposées. Il faudrait être particulièrement malintentionné pour concevoir, au fil de la lecture de ces pages bien argumentées et convaincantes, un doute quelconque quant à la pertinence de l'étalon utilisé, constitué pour une grande partie de textes du XIII ${ }^{e}$ siècle. Mais on se demande par moments si la comparaison avec les romans arthuriens cycliques, Huon de Bordeaux ou les Voeux du Paon, pourtant tous d'indéniables avant-textes, est suffisante pour «expliquer» ce qu'on a dans Ysaie. On peut se demander en effet s'il ne faudrait pas mobiliser, en plus de la tradition littéraire antérieure, quelques textes qui appartiennent plus spécifiquement au Moyen Age finissant. Personnellement, ce doute m'a affleuré au moment de lire les remarques de Patricia Victorin à propos du portrait du héros, qui est présenté ainsi: Moult fu Ysaÿe plains de grant biauté, fors, grans, apers et hardis par samblanche. Et ot .IX. piés de loing et s'estoit cras et membrus a l'avenant, les cheveulx noirs et creppés et les yeulx vairs, grande bouche et rousse barbe, les bras plas et ossus et puins quarrés et jambe vautiche, et avoit sy crueux regart c'a mervelles, et non pourquant rioit volentiers par nature (Ysaie, éd. Giacchetti, $\S 27)$. Patricia Victorin s'interroge à juste titre sur la rousseur de la barbe et les traits de caractère négatifs mentionnés, peu fréquents dans les portraits des héros romanesques. Elle suggère que le héros est dépeint comme un «surmâle», voire une «brute épaisse» (p. 232), très nettement en retrait par rapport aux Lancelot et Perceval de la tradition antérieure. Il est vrai qu'au Moyen Age, les cheveux roux sont en général mal vus et que les protagonistes sont souvent présentés comme uniformément positifs, mais il suffit de regarder, pour trouver des descriptions assez semblables au portrait d'Ysaïe, et dépourvues de toutes connotation négative, celles que fournissent les armoriaux arthuriens, évoqués par Patricia Victorin dans un autre contexte. Ces près de 180 descriptions qu'ils contiennent constituent sans doute le corpus le plus important de 
portraits, arthuriens ou autres, que nous ait légué le Moyen Age et ont l'avantage d'être plus ou moins contemporaines d'Ysaïe. Or, dans les descriptions des armoriaux auxquelles ressemble à s'y méprendre le portrait d'Ysaïe par sa facture - un grand nombre de personnages - et non des moindres - ont les cheveux et la barbe de la même couleur qu'Ysaïe, et le «mélange» de qualités y est constant. A propos du Chevalier de Norgalles, on y lit par exemple: Il estoit grant de corps, bel et droit a merveilles, les cheveux eust noirs, les yeulx roux et etincellans et moult avoit fier regart, barbe eust rousse et la coulleur $d u$ visage ung peu palle. Les espaulles eust belles et grandes, les bras longz et nervuz, les pointz eust gros et carrés. Par le cas n 'estoit pas trop ingre, les cuisses eust grosses et longues et les jambes moult bien faictes [...]. De son corps estoit liger et si asseuré que oncques nul ne le fut plus. Large et liberal estoit, poy aimoit chasse ne autre deduit. Quant il estoit courroucé il devenoit tout blanc, trambloit et faisoit parler ses de[n]s comme ung sanglier. (cité d'après le ms Arsenal 4976, fol. $28 \mathrm{v}^{\circ}$ ). On peut, dans ces armoriaux, trouver d'autres descriptions où les cheveux roux et les traits de caractères non uniformément positifs apparaissent conjointement ou séparés. Si le roux paraît être neutre dans les armoriaux, la raison en est probablement que l'aune à laquelle se mesure et s'évalue le trait n'est là pas «littéraire», mais bien réelle: dans une société où les cheveux roux ne sont pas rares nous sommes selon toute vraisemblance dans le nord de la France, non loin de la terre d'où est originaire Johnny Halliday, et assez loin du monde méditerranéen de la Bible il ne peut être question de stigmatiser de la sorte une partie importante de la population. Quant aux mauvaises teches, ce sont les «détails qui ne s'inventent pas» et ils font désormais partie du portrait d'un personnage. Les canons du portrait littéraire ont visiblement changé et la comparaison avec les descriptions d'un Lancelot ou d'un Tristan ne rend qu'imparfaitement compte des nouveaux paramètres. De façon plus générale, l'esthétique qui gouverne Ysaïe est probablement déjà assez éloignée de celle, par exemple, des romans arthuriens en prose, avec lesquels il partage pourtant aussi ostensiblement un grand nombre de traits. On peut même se demander si Ysaie est encore un roman «médiéval». Ainsi, le lecteur qui vient du XII ${ }^{e}$ et $\mathrm{XIII}^{\mathrm{e}}$ siècle va certes frémir devant l'idée saugrenue de doter Tristan et Yseut d'une descendance, mais le lecteur familier des imprimés la trouvera plus normale. On connaît en effet, en espagnol, Tristán de Leonis y el rey don Tristán el joven, su hijo, imprimé à Séville en 1534, dont toute la seconde partie est dédiée à Tristan iunior, et qui a été traduit en italien et imprimé à Venise en 1555. L'adynaton n'existe donc que pour le médiéviste et non pour le public du XVI ${ }^{e}$ siècle; qui s'occupe d'incunables et d'imprimés peut dresser toute une série d'oeuvres, qui, comme Ysaïe, imprimé lui-même à quatre reprises et conservé dans deux manuscrits dont l'un paraît dater du $\mathrm{XVI}^{\mathrm{e}}$ siècle, mettent en scène des pères ou fils de personnages célèbres. C'est devant cet horizon qu'il convient de situer aussi Guiron le Courtois, imprimé plusieurs fois en français et en italien et traduit en vers italiens pour François $\mathrm{I}^{\mathrm{er}}$. Le domaine épique n'est pas non plus en reste avec les «romans» comme Meurvin et Mabrian qui ont également pour héros des fils de personnages renommés. A chaque fois, la démarche consiste à créer des protagonistes neufs qui ne soient pas des «inconnus», puisqu'ils ont pour pères ou pour fils de héros célèbres; ils sont archiconnus, mais «frais», unverbraucht. Ce qui est vrai pour les personnages l'est sans doute aussi pour l'univers romanesque dans lequel ils évoluent. Ces textes de la fin du Moyen Age obéissent à une esthétique qui n'est plus celle du XIII siècle, si bien qu'Ysaïe est sans doute aussi proche de l'Amadis que du Lancelot en prose. Il faudra un jour procéder à une coupe synchronique à travers la production narrative de la fin du Moyen Age et du début de la Renaissance pour dégager les paramètres de cette 
production narrative. Ysaïe y aura toute sa place; pour tout l'héritage «médiéval» que comporte le roman, Patricia Victorin nous offre ici une magistrale étude qui comble de façon heureuse une vraie lacune. 